\title{
Bottom-up modeling of residential batteries and their effect on system-level generation cost
}

DOI:

10.1016/j.epsr.2020.106711

\section{Document Version}

Accepted author manuscript

Link to publication record in Manchester Research Explorer

\section{Citation for published version (APA):}

Jaglal, D., Procopiou, A. T., Petrou, K., \& Ochoa, L. F. (2020). Bottom-up modeling of residential batteries and their effect on system-level generation cost. Electric Power Systems Research, 189, 106711.

https://doi.org/10.1016/j.epsr.2020.106711

\section{Published in:}

Electric Power Systems Research

\section{Citing this paper}

Please note that where the full-text provided on Manchester Research Explorer is the Author Accepted Manuscript or Proof version this may differ from the final Published version. If citing, it is advised that you check and use the publisher's definitive version.

\section{General rights}

Copyright and moral rights for the publications made accessible in the Research Explorer are retained by the authors and/or other copyright owners and it is a condition of accessing publications that users recognise and abide by the legal requirements associated with these rights.

\section{Takedown policy}

If you believe that this document breaches copyright please refer to the University of Manchester's Takedown Procedures [http://man.ac.uk/04Y6Bo] or contact uml.scholarlycommunications@manchester.ac.uk providing relevant details, so we can investigate your claim.

\section{OPEN ACCESS}




\title{
Bottom-up Modeling of Residential Batteries and their Effect on System-Level Generation Cost
}

\author{
Dillon Jaglal $^{1}$, Andreas T. Procopiou ${ }^{1}$, Kyriacos Petrou ${ }^{1}$, Luis F. Ochoa ${ }^{1,2}$ \\ 1 The University of Melbourne, Melbourne, Australia \\ 2 The University of Manchester, Manchester, United Kingdom
}

\begin{abstract}
The declining cost of residential battery energy storage (BES) and photovoltaic (PV) systems enable customers to significantly reduce their energy dependency from the grid. By absorbing excess PV generation, BES systems could reduce impacts associated with the 'duck curve'. However, since off-theshelf (OTS) BES systems are controlled for customer benefits, this absorption might be limited at times, resulting in similar duck curve effects. This work proposes a bottom-up modelling approach to demonstrate the system-level generation cost effects from residential BES systems. Using a modified IEEE 9-bus system with real anonymized smart meter data, results show that with the OTS BES control, the overall reduction in system net demand due to peak PV generation still occurs which leads to baseload generation shutting off, incurring additional costs. Alternatively, the adoption of smarter BES controls aimed at mitigating impacts on distribution networks by reducing PV exports can bring significant system-level generation cost reductions.
\end{abstract}

Index Terms-Battery Energy Storage Systems, PV Impacts, PV Systems, Residential Customers, Unit Commitment.

\section{INTRODUCTION}

PV installation costs have reduced significantly in recent years and adoption rates have been rapidly increasing. While a more decentralized and low-carbon electricity system is desirable for many countries, large PV penetrations can significantly reduce the distribution network demand seen at the system level. Indeed, the effects of PV generation on net demand creates the well-known 'duck curve' [1] which can affect the behavior of conventional generating units (e.g., due to generation displacement or ramping needs) and, in turn, the overall system cost [2]. Australia, for example, has seen almost a 50\% increase in cumulative solar PV capacity within a year, reaching $12 \mathrm{GW}$ in 2019 from $8 \mathrm{GW}$ in 2018. Similar 'duck curve' effects are already becoming evident due to large demand reductions seen at the system level [3].

As cost reduces and adoption trends increase for residential battery energy storage (BES) systems, customers will be able to make better use of their existing PV systems, further reducing their electricity bills. Since BES systems are effective in increasing self-consumption, there is an expectation that the widespread adoption of BES systems will inherently help reduce the reverse power flows and, hence, mitigate the impacts associated with the duck curve (particularly around midday) on the power system [4]. However, existing self-consumption controllers embedded in commercially available BES systems, hereafter referred to as off-the-shelf (OTS), are ineffective in this matter providing little to no benefits to reverse power flow reduction. As demonstrated in [5], on a sunny day with no one at home, OTS BES systems start charging from the excess PV generation relatively early in the morning, quickly reaching full state-of-charge (SOC) even before high levels of PV generation occur. Consequently, given that the OTS BES system cannot store more PV generation after that time, the excess PV generation will be fed back to the electricity network. Hence, similar issues related to large residential PV penetrations may still occur even when coupled with BES systems.

The effects of changing demand patterns on system-level cost have been investigated in the literature by mainly considering active, price-driven controls of demand, PV systems and BES systems. Using the unit commitment (UC) to assess the system-level cost, in [6] and [7], aggregated demand was considered to actively respond to prices. In [8], a bi-level optimization strategy is used to minimize system-level cost involving the UC whilst maximizing aggregator/customer benefits by controlling aggregated PV and BES systems. Similarly, a cost minimization approach was proposed in [9] that reduces information exchange which requires a central entity to set price signals for prosumers to respond to. While these types of studies provide insights of how the system can benefit from actively controlling behind-the-meter demand and system generation, such level of flexibility is unlikely to be available in the near future. On the other hand, when the system level effects are considered with the adoption of residential PV and BES systems (OTS) such as in [10], system-level cost savings are reported. However, [10] assumes that households with PV only or with a PV/BES system will not export into the grid at all times. As shown in [5], both these types of customers can export large amounts of PV generation into the grid, and therefore the effect that this can have at a system level as PV penetration increases needs to be assessed. This can lead to an underestimation of system costs as PV penetration increases; due to aggregated exports displacing baseload generation during midday. 
Furthermore, it may be very useful for network operators and policy makers to first understand the effects that OTS BES systems and the resulting reduction in system net demand will have on system-level cost. A main contribution of this work is to assess what cost implications the growing adoption of residential OTS BES systems will have on system-level generation cost, adequately considering the interactions of the technologies (PV and BES systems) and demand.

This work assesses the extent to which the widespread adoption of OTS BES systems can affect system-level generation costs using a UC. To achieve this, system-level net demand profiles are produced using a bottom-up approach for the residential component. Real anonymized smart meter data are adopted to produce net demand profiles for individual households. The UC is performed on the resulting system-level net demand and then the impacts are analyzed. For comparison purposes, a PV-only scenario is used as the baseline. Furthermore, to quantify the potential system-level benefits from a smarter, decentralized BES controller, such as the one proposed in [11] (designed to provide local network benefits by reducing PV exports) is also investigated. The analysis is performed on a modified IEEE 9-bus system.

\section{OVERVIEW OF OPERATING SCHEMES AND PROFILES}

Refer to Fig. 1 for an illustration of the behavior of the PV and BES systems at the household-level for each of the three operation schemes under assessment. Fig.1 (a) shows only the load demand and the clear-sky irradiance of the PV systems. Each of the following plots (Fig. 1 (b-d)) are as follows:

PV Only. This scheme considers the case where only PV systems are available in the system and corresponds to the BAU scenario to observe the behavior of the UC under different PV penetrations. As seen in Fig.1 (b) during high PV generation hours, the household net profile results in large exports, $P_{P V}^{\exp }$ due to the large PV generation and small load demand. As mentioned before, this can bring technical challenges not only to the operation of the distribution network (e.g., voltage rise) but also to the whole system (due to the 'duck curve').

Off-the-shelf (OTS). This scheme considers the case where OTS BES systems are installed along with the PV systems. The OTS control is based on what manufacturers provide as general description of the basic operating principles and corresponds to the following: when generation exceeds demand, the BES system charges from all the surplus PV generation. When PV generation falls below the household demand, the BES discharges to meet the local demand [5]. This is demonstrated in Fig. 1 (c) where all the excess of PV generation is being stored in the BES system until it becomes full (i.e., full SOC). After that point, any excess of PV generation is exported back to the grid (here, the maximum exported power is denoted as $P_{O T S}^{\exp }$ ) until local demand exceeds generation, to which point the BES system discharges to meet the local demand. Since the BES systems can reach full SOC quite early, the peak exported power can be virtually the same as with the case of PV Only $\left(P_{\text {OTS }}^{\text {exp }} \approx P_{P V}^{\text {exp }}\right)$. In practice, this can mean large exports at times
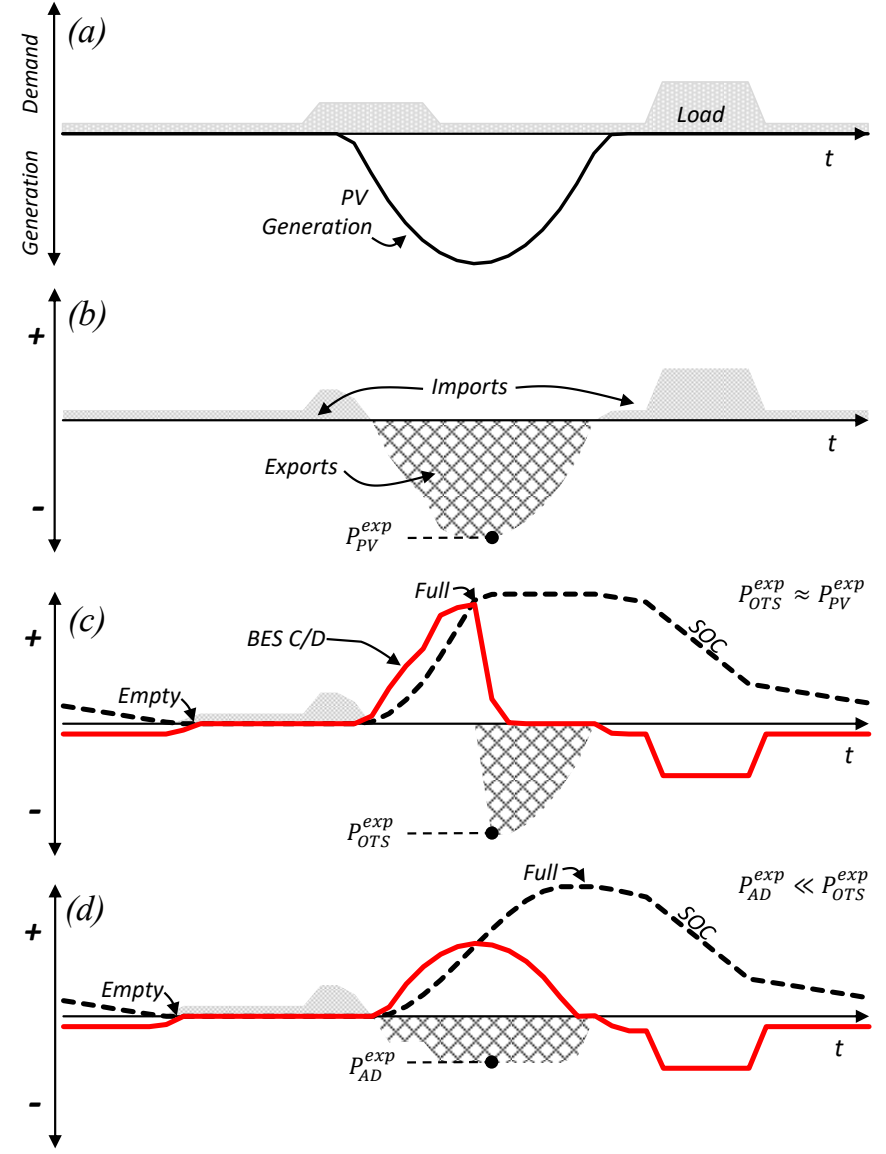

Figure 1. Household-level illustration of the operation considering different operating schemes

of high PV generation, resulting in similar challenges as the case without BES systems (PV Only).

Adaptive Decentralized (AD) Control. This scheme considers the case where BES systems adopt a 'smarter' controller [11], designed to overcome the limitations of the OTS control (BES systems reaching full SOC very early; hence inadequate to reduce reverse power flow). The $\mathrm{AD}$ control reduces high $\mathrm{PV}$ exports by adapting the BES charging power proportionally to the PV generation, and ensuring available capacity by discharging overnight. The design allows it to adapt in real time changes such as cloud transients and household demand. Fig.1 (d) illustrates this operation where PV exports (here, the exported power is denoted as $P_{A D}^{\exp }$ ) can be significantly reduced while meeting the local needs of the household. This, in turn, means that the integrity of the distribution network is not compromised as the magnitude of the reverse power is significantly reduced $\left(P_{A D}^{\exp } \ll P_{O T S}^{\text {exp }}\right)$. Consequently, for the whole system, the effect on the midday net demand will be much less, potentially reducing the displacement of conventional generating units and ramping needs.

\section{METHODOLOGY}

The adopted approach to assess the effects of PV Only, OTS and the AD control schemes on the system level cost is 
presented in this section. As depicted in Fig.2, first, for each operation scheme and for a given PV and BES system penetration, the net demand profiles (residential and nonresidential) are produced adopting a bottom-up approach. Then, the resulting net demand profiles are incorporated to the unit commitment (UC) and the corresponding UC outputs are stored. The process is repeated for the predefined penetration levels and, finally, all results are used to analyze and compare key parameters among the three operating schemes.

\section{A. Bottom-Up Production of Net Demand Profiles}

For residential customers, the set $I$ is defined as customers with demand only (no PV or BES systems). Customers with PV systems belong to the set $P$ and customers with PV and BES systems belong to the set $B$, with indices $i, p$ and $b$, respectively. For a given time horizon, defined as set $T$ and indexed by $t$, the demand of a residential customer with demand only, is defined as $P_{i, t}^{D}$. The net demand for residential customers with PV systems, $P_{p, t}^{D P V}$, is calculated using (1) where $P_{p, t}^{P V}$ and $P_{p, t}^{D}$ correspond to the $\mathrm{PV}$ and demand profiles, respectively. For customers with PV and BES systems, the net demand, $P_{b, t}^{D B E S}$, is determined by the summation of household demand, $P_{b, t}^{D}$, PV generation, $P_{b, t}^{P V}$, and the power of the BES system, $P_{b, t}^{B E S}$ as shown in (2). $P_{b, t}^{B E S}$ is the result of the operation of the battery using a given scheme, namely OTS or AD control. The power of the BES can be positive or negative denoting charging or discharging respectively.

Non-residential customers belong to the set $K$ with index $k$ and the demand is defined as $P_{k, t}^{D N R}$. The summation of each term of residential customers (elements from $I, P$, and $B$ ) and the term with non-residential customers (element from $K$ ) are used to calculate the system-level net demand, $P_{t}^{D S L}$, as in (3).

$$
\begin{gathered}
P_{p, t}^{D P V}=P_{p, t}^{D}-P_{p, t}^{P V} \quad \forall p \in P, \forall t \in T \\
P_{b, t}^{D B E S}=P_{b, t}^{D}-P_{b, t}^{P V}+P_{b, t}^{B E S} \quad \forall b \in B, \forall t \in T \\
P_{t}^{D S L}=\sum_{i \in I} P_{i, t}^{D}+\sum_{p \in P} P_{p, t}^{D P V}+\sum_{b \in B} P_{b, t}^{D B E S}+\sum_{k \in K} P_{k, t}^{D N R} \\
\forall t \in T
\end{gathered}
$$

$P_{t}^{D S L}$ forms the input to the UC which quantifies the commitment of generating units giving the dispatch of the generating units to satisfy the system-level demand across $T$.

\section{B. Unit Comittment}

The system level impacts are quantified using a UC based on the formulation presented in [12] with the use of quadratic cost functions making it a mixed integer quadratic problem (MIQP). The objective function is to minimize the total cost of generation (set of $J$ generating units indexed by $j$ ) which comprises of production cost, $c_{j t}^{P}$ startup costs, $c_{j t}^{u}$ and shutdown costs, $c_{j t}^{d}$ as shown in (4) to satisfy the system-level

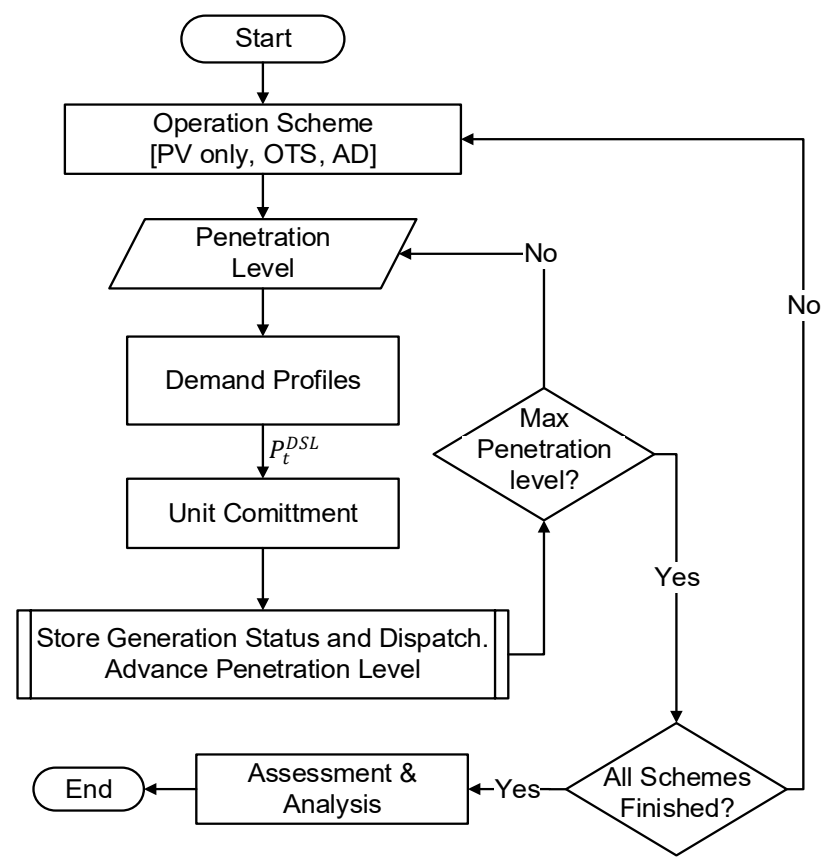

Figure 2. Overview methodology to assess operation schemes

TABLE I. GENERATION PARAMETERS USED FOR THE UC

\begin{tabular}{c|c|c|c|c|c} 
Generating Unit & Fuel Type & $\begin{array}{c}\text { Rating } \\
\text { (MVA) }\end{array}$ & $\begin{array}{c}\text { MSG } \\
\text { (MW) }\end{array}$ & $\begin{array}{c}\text { UT min. } \\
\text { (h) }\end{array}$ & $\begin{array}{c}\text { DT min. } \\
\text { (h) }\end{array}$ \\
\hline \hline U1-U6 & Coal & 51.4 & 7.89 & 20 & 18 \\
\hline U7-U8 & OCGT & 50.0 & 7.4 & 2 & 1 \\
\hline U9-U14 & OCGT & 31.4 & 5.2 & 1 & 1
\end{tabular}

demand $P_{t}^{D S L} \forall t \in T$. This gives the power dispatch for each of the generating units in $J$, across the horizon $T$, that minimizes the total cost and satisfies $P_{t}^{D S L}$.

$$
\text { Minimize } \sum_{j \in J} \sum_{t \in T} c_{j, t}^{P}+c_{j, t}^{u}+c_{j, t}^{d}
$$

The UC considers the following constraints: power balance, spinning reserves, maximum ramping capability (increasing and decreasing of active power), minimum stable generation (MSG) and minimum up and down times. The full formulation can be found in [12]. A UC is run for each operating scheme and penetration level and the corresponding outcomes (i.e., generation status, the dispatch power and total cost) are stored to then be used for the assessment and analysis.

\section{Assessment of System Level Impacts}

Once the UC for each of the predefined PV and BES penetration levels and operation schemes is completed, the corresponding effects of each penetration level has on the system are assessed considering the following:

1) Generating Units Power Dispatch: The time-series power dispatch of each generating unit is plotted as a stacked area chart. This allows to visualize the impact that each control scheme has on the behavior of conventional generating units 
(e.g., ramping, magnitude of power dispatch for each $t$, and displacement).

2) Total Cost of Generation: This is the cost across the time horizon $T$ for each operation scheme and penetration level. It comprises of fuel costs (production of energy), startup costs and shutdown costs. This allows the system operator to estimate the overall cost across a given horizon and better understand cost projections as penetration levels increase.

3) Average Cost per $M W h$ : Defined as the average cost per megawatt-hour of conventional energy generated. This cost is averaged across online generators from $J$, throughout the time horizon $T$. This metric will capture the effects of higher penetrations (e.g., ramping, startup and shutdown costs) on the price of energy.

\section{CASE STUdY}

In this section, the methodology is applied for the three operation schemes shown in Section II. Firstly, all case study considerations (i.e., set of generating units, parameters for the UC, net demand profiles etc.) are presented. Then, the net demand profiles for each operation scheme and penetration are produced and provided. Lastly, the system-level impacts are presented and discussed. The optimization formulation is performed using Julia [11] considering a mixed-integer quadratic program (MIQP) solved using Gurobi 8 [12].

\section{A. Case Study Considerations}

The set of generating units used for the UC corresponds to the modified IEEE 9-bus test system used in [13] and [14]. The generation fleet comprises of 14 generators: six baseload generating units represented as coal-fired generation and eight flexible peaking units represented as gas-fired generation. Key parameters used for the analysis are shown in Table I with the ratings (i.e., MSG), minimum up time (i.e., UT) and minimum down time (i.e., DT). A spinning reserve of $36 \mathrm{MW}$ is adopted, as this is sufficiently large to cope with the largest generation contingency [15]. All generation parameters used for the modelling are adopted from [16], [17] and [18].

The total amount of residential customers used in this case study was based on the methodology proposed by the authors in [13] and [14]. Normalized residential and non-residential (i.e., commercial) profiles were scaled according to a 28.6 and $71.4 \%$ energy mix, respectively (2014-2015 Australian total energy consumption values [19]). This superimposed profile was then scaled to achieve the system peak demand of the modified IEEE 9-bus test system. By multiplying the total energy of the resulting system profile with the residential energy proportion (i.e., 28.6\%), and then dividing by the energy of a single normalized residential profile, the total number of residential customers was derived: 73,440 . It should be noted that the normalized residential profile was used only for the purposes of deriving the number of residential customers.

Once the number of residential profiles is defined, individual residential profiles for each type of residential customer are created based on the methodology presented in
Section III-A. For the demand profiles (i.e., $P^{D}$ ) a pool of $30-$ min resolution Australian smart meter demand data (from 2014, provided by AusNet Services) is used. The PV profiles (i.e., $P^{P V}$ ) correspond to a real clear-sky irradiance profile from the same region, multiplied by the PV system size of the household. A different size of PV system is allocated to each customer; which is based on Australian installation statistics from 2016 onwards [20] with capacities of $2.5,3.5,5.5$ and $8 \mathrm{~kW}$ and distributions of $10,30,50$, and $10 \%$ respectively.

The BES profile (i.e., $P^{B E S}$ ) is dependent on the demand and PV profile of each customer. For each customer, the methodology found in [5] was used to define the BES profile for the OTS control, and in [11] for the AD control. The BES systems are sized at $5 \mathrm{~kW} / 13.5 \mathrm{kWh}$ with $100 \%$ depth of discharge and $88 \%$ roundtrip efficiency. This BES is selected as it is widely available for customer use in the Australian market [21].

For this case study, the three operation schemes are investigated for penetration levels from $0-100 \%$ in $10 \%$ intervals. Penetration level in this work represents the percentage of residential customers with $\mathrm{PV}$ or/and BES systems. This gives a total of $3 \times 11$ scenarios $(0 \%$ inclusive to give 11 penetration levels). As penetration levels increase, load growth is considered as static for each operating scheme. Using the load, PV and BES information previously presented, system level demands are developed to be used in the UC.

The $\mathrm{UC}$ is run at $30 \mathrm{~min}$ intervals for a 24 -hour time horizon for a typical clear-sky summer day.

\section{B. Net Demand Profiles}

Considering the effects of PV systems, these can be observed in Fig. 3 where the $0 \%$ penetration level yields a peak system demand of $335.08 \mathrm{MW}$. The net demand is reduced with higher PV generations, and as expected, the maximum reduction occurs around midday when generation is at the highest. The corresponding demand reduction continues as PV penetration increases from 0 to $100 \%$ with $100 \% \mathrm{PV}$ penetration having a maximum reduced net demand of close to zero.

With the OTS BES systems, during the off-peak hours the net demand is slightly reduced. This is due to the control strategy of the OTS scheme where the household demand is being supplied locally by the stored energy from the batteries. During the initial PV generation hours (8:00-10:00) most of the excess PV is used to charge the batteries until around midday. Around this time the batteries become full and the excess PV is exported to the grid, this is seen as the reduced net demand. The large reverse power flows from the PV systems are slightly time-shifted and the maximum reduction of net demand is virtually unchanged for the case of the OTS. This trend continues for increasing penetrations.

For the case of BES systems with the AD control, it is observed that the innate by-product of this control is such that the net demand profiles have a flatter shape. This effect as briefly described in Section II-A is due to the smarter charging operation of the batteries in a way that the reverse power flow 

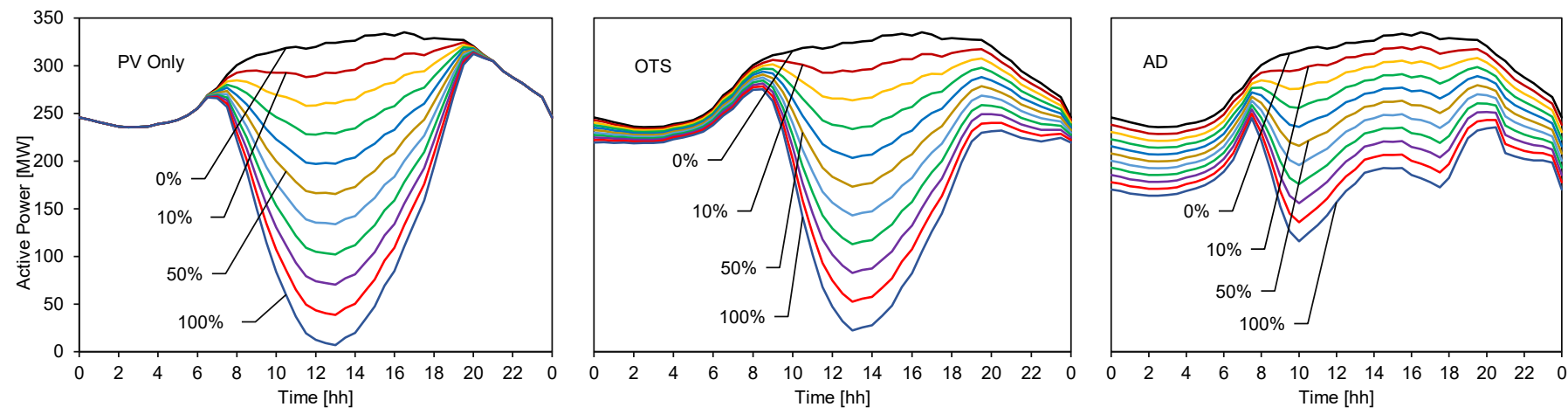

Figure 3. System level net demands: PV Only, OTS, AD.
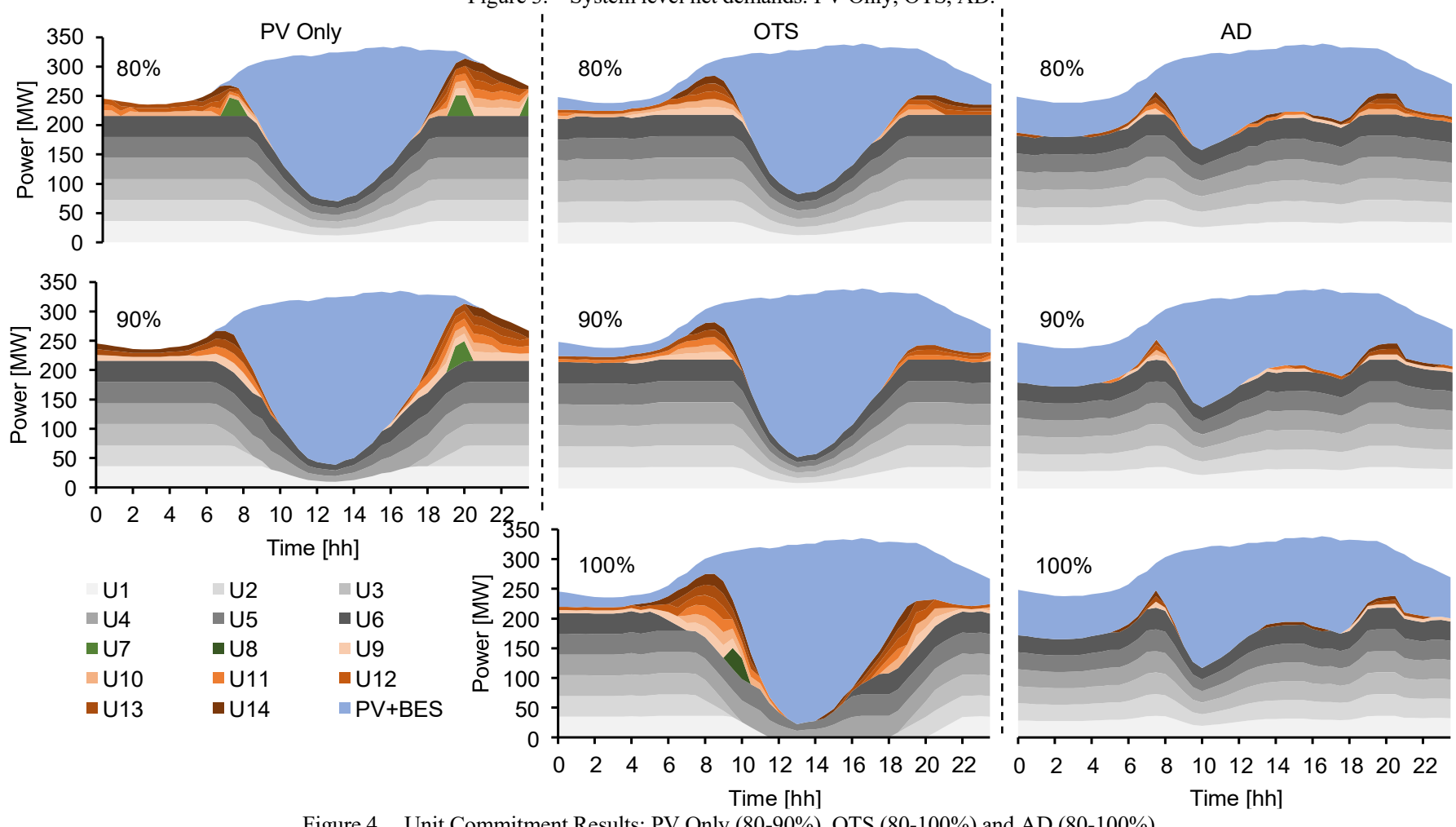

Figure 4. Unit Commitment Results: PV Only (80-90\%), OTS (80-100\%) and AD (80-100\%).

(due to excess PV generation) are significantly reduced compared to the case of the OTS. Furthermore, the discharging operation of the $\mathrm{AD}$ which ensures that enough capacity is available for the next charging period is further reducing the system profile compared to the OTS during off-peak hours. This reduction is expected to diminish the need for gas generation which brings overall cost benefits.

From the perspective of the system, a flatter demand profile may be considered an attractive behavior as it potentially leads to less aggressive ramping, starting and stopping requirements of thermal generators. Furthermore, this might entail less dependency on oversized assets required to cope with shortterm peaks/spikes in demand. On this front, the system-level impacts of the aforementioned demand profiles are examined in the following section.

\section{System-Level Impacts}

As PV penetration increases, the system-level net demand reduces (as expected). The effect on the set of generating units and their outputs is seen in Fig. 4 showing the results from the UC from $80-100 \%$ penetration only. The UC results for the lower penetrations $(0-70 \%)$ are excluded since the incremental effects on the scheduling of generators do not lead to significant displacement of generating units. However, the energy and cost results are presented later (Fig. 5 to Fig. 7). As PV penetration continues to increase for PV only, the net demand reduces to the extent where the MSG of baseload generation becomes constrained leading to a non-feasible solution at $100 \%$ penetration as plants are not able to ramp further down; hence, no results are presented. When batteries are adopted, with either control scheme, generating units are required to provide more output during high PV generation hours. Thus, for those cases, feasible solutions exist at $100 \%$ penetration. 
Compared to the case of PV only, OTS results in less gas generation required during evening hours. This is due to the BES systems supplying the local load demand which have slight cost benefits which is discussed later. When looking at the behavior of the conventional plants during peak PV generation, it can be seen that for PV Only and OTS the online generating units are very similar.

For the $\mathrm{AD}$ scheme, the unit commitment results take on a very different behavior. None of the baseload generating units are switched off and the ramping requirements appear to be very gradual (reduced slope of power dispatches between time steps). To further understand what this means, the energy and cost parameters should be considered.

Fig. 5 shows the contributions of energy from the two technology types that were modelled, gas and coal. As PV penetrations increase, the energy of both coal and gas-fired generation are reduced. However, for high penetration levels the OTS and AD schemes result in more coal and less gas generation compared to $\mathrm{PV}$ only, with the $\mathrm{AD}$ scheme requiring the lowest amount of gas generation while utilizing more coal generation. The $\mathrm{AD}$ scheme allows more baseload plants to be on (i.e., the inflexible yet cheap units) and relies less on peaking plants (i.e., flexible but more expensive). This idea is further demonstrated by the reduction in the amount of energy required from gas generation being effectively reduced as PV penetrations increase. Again, reducing the amount of more expensive, flexible gas generation.

As previously mentioned, the $\mathrm{AD}$ control has the effect of increasing demand at peak $\mathrm{PV}$ generation periods thus requiring more gas outputs. This means that in the short term, AD will result in slight increases of overall costs due to this effect for 0 to $50 \%$ penetration as seen in Fig. 6 . However, the net effect of reducing the demand during off-peak periods, significantly reduces the need for gas generation and results in overall cost reductions for cases of $\mathrm{PV}$ penetration exceeding 50\%. This trend continues for all cases until $100 \%$ penetration levels. It follows that there can be an overall generation cost reduction from widespread adoption of BES systems with the AD control.

When looking at the average cost per megawatt-hour of conventional energy generated for the PV Only scheme in Fig. 7, there is a reversal trend (increased costs) onwards from $60 \% \mathrm{PV}$ penetration. This is seen since the amount of energy displaced by PV results in no additional cost benefit for high PV penetrations due to the expense of other operational costs of conventional generating units (startup and shutdown costs). As was the case for total cost, the AD control has the lowest average cost per megawatt-hour when compared to both PV only and OTS for penetrations exceeding 50\%.

There was a notable deviation in the energy and cost curves for the OTS case of $100 \%$ penetration. Here, OTS is more expensive because of the technical constraints of the generators. When baseload generation switches off, the minimum downtime of the coal plants forces more expensive gas generators to fill the demand during 16:00-19:00 (relate to Fig. 4) which exacerbates the costs. The cost is further increased by incurring the startup and shutdown costs of the larger, slower

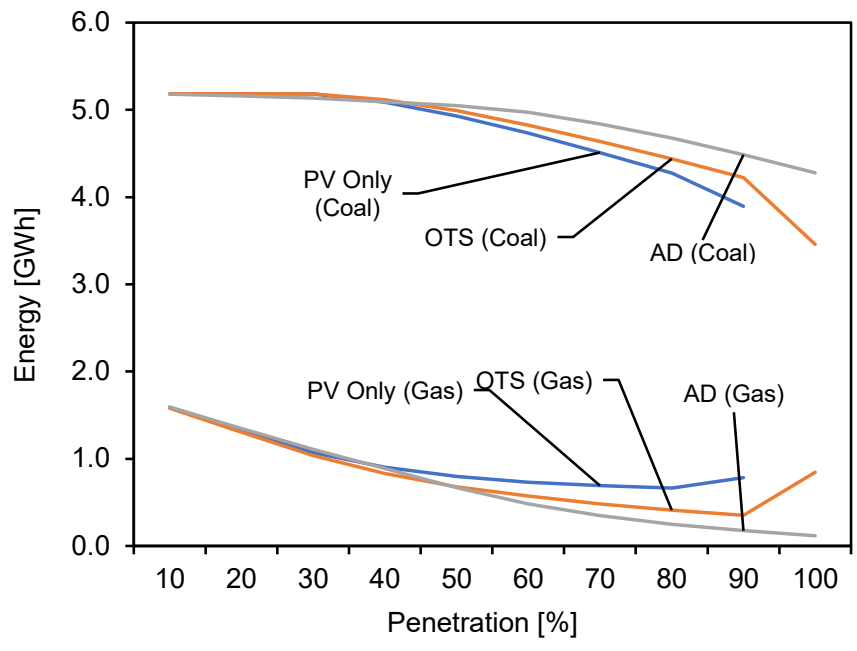

Figure 5. Energy outputs from Coal and Gas Generation

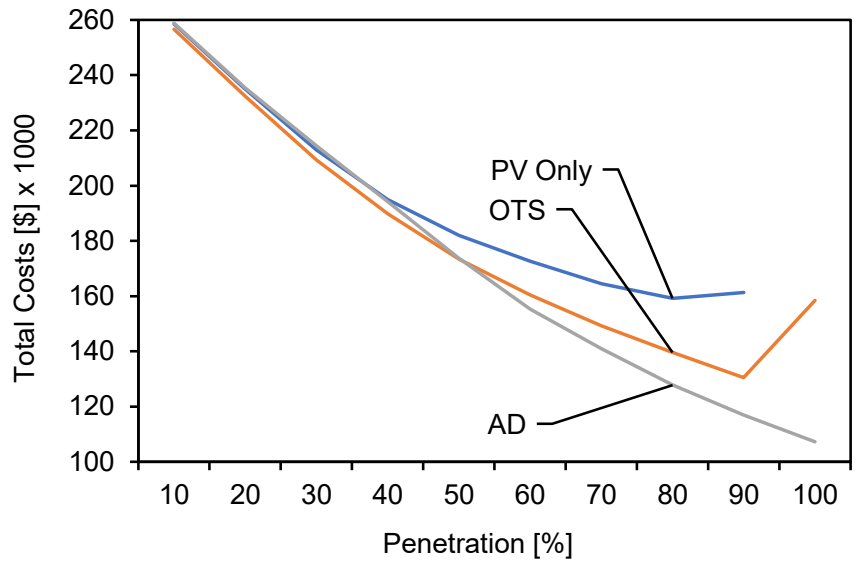

Figure 6. Total generation cost vs penetration

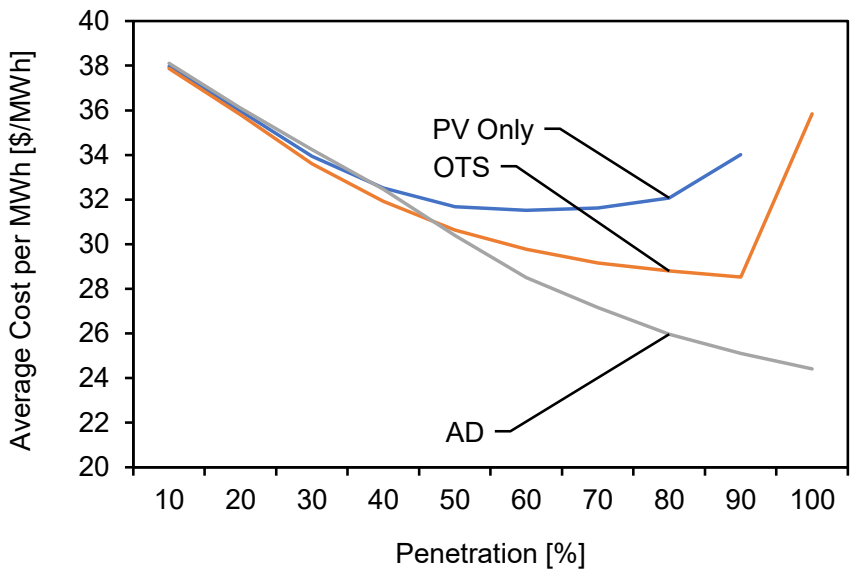

Figure 7. Energy cost vs penetration

coal plants when they are turned back on later in the evening hours. This behavior is reflected in both the energy and utilization factors as reduced coal and increased gas usage. For the costs, a sharp reversal trend is seen with average cost per megawatt-hour reaching as high as the costs seen for the $20 \%$ PV penetration case. 
A very interesting finding of this case study is that while the total system cost reduces the more residential PV and OTS BES systems are adopted, the average cost of generation per MWh of conventional generation increases around the $50 \%$ penetration level. Taken into consideration that now more than half of the (residential) customers are mostly self-sufficient, this could lead to an increase in electricity prices for customers without local generation. The adoption of more baseloadfriendly BES controls such as the AD manage to further reduce the average cost of conventional generation per MWh, even past the $50 \%$ penetration level. Therefore, the adoption of such systems could prove to not only provide benefits to their owners, but for everyone in the power system. While the case study presented in this work is highly realistic, additional analyses that consider real systems need to be conducted to quantify the full range of impacts and benefits.

\section{DISCUSSION}

This work highlights the potential impacts to the system level if residential OTS BES systems are adopted on a large scale and provides an understanding on how these impacts can be mitigated with smarter BES controllers. However, the case study performed in this work considers only a single day analysis (typical clear-sky summer day). Longer time-horizon analyses may be adopted to capture intertemporal and seasonal changes that may occur. Similarly, the adoption of PV and BES systems will progress with time and so load patterns may evolve (e.g., load growth due to population, non-residential PV systems or new technologies such as electric vehicles), which could also be catered for in longer time horizons. Furthermore, PV and BES systems may also lead to changes on market prices/generation costs (e.g., due to changing load behavior). Further work can be performed to capture these effects.

\section{CONCLUSIONS}

This work investigated the effects that the widespread adoption of commercially available off-the-shelf (OTS) residential battery energy storage (BES) systems might have on the system-level generation cost. Potential future scenarios of different penetration levels of photovoltaic (PV) and OTS BES systems are examined using a unit commitment. For comparison purposes, a PV-only scenario is used as the baseline. Furthermore, the system-level benefits from a 'smarter', decentralized BES controller, designed to provide local network benefit by reducing PV exports such as the one proposed in [11] is also investigated (referred to as the adaptive decentralized (AD) control). System-level net demand profiles (residential and non-residential) are produced using a bottomup approach from real anonymized smart meter data of individual households.

Results show that, with the OTS controller, the net exported power from residential customers are comparably as large as customers with PV only (i.e., no BES systems). With OTS slightly reducing the need for flexible gas generation, this results in marginal short-term cost benefits. However, the large exported power during high PV generation hours results in increased start/stop operation of conventional generating units (similar to the case of PV only) which potentially increases cost and introduce additional challenges in the long term.

On the other hand, this work highlights notable improvement in cost can be achieved by adopting smarter controllers, such as the one investigated in this paper. As observed, the AD control significantly reduces the PV exports, bringing overall cost benefits to the system for high penetrations. This is due to generating units operating with less aggressive ramps, to meet changes in demand, together with reduced start/stop operation and overall reduction for the need of flexible gas generation.

The resulting effect of reduced cost of conventional generation for the AD control scheme may be particularly attractive since it not only reduces generating costs but also brings benefits to the distribution network and residential customers.

This work can be useful for system operators to be pragmatic in planning by providing a deeper understanding of the future implications of the widespread adoption of BES systems. This can ultimately lead to more effective coordinating efforts between the system operator and distribution companies.

\section{REFERENCES}

[1] P. Denholm and R. Margolis, "Energy Storage Requirements for Achieving 50\% Solar Photovoltaic Energy Penetration in California," National Renewable Energy Laboratory, Golden, CO, 2016.

[2] D. Lew and N. Miller, "Reaching new solar heights: integrating high penetrations of PV into the power system," IET Renewable Power Generation, vol. 11, no. 1, pp. 20-26, 2017.

[3] Market Insights \& WA Market Operations, "Quarterly Energy Dynamics - Q1 2019," AEMO, 2019.

[4] IRENA, "Electricity Storage and Renewables: Costs and Markets to 2030," International Renewable Energy Agency, Abu Dhabi, 2017.

[5] K. Petrou, L. F. Ochoa and A. T. Procopiou, "Limitations of Residential Storage in PV-Rich Distribution Networks: An Australian Case Study," in 2018 IEEE Power \& Energy Society General Meeting (PESGM), Portland, OR, 2018.

[6] A. Khodaei, M. Shahidehpour and S. Bahramirad, "SCUC With Hourly Demand Response Considering Intertemporal Load Characteristics," IEEE Transactions on Smart Grid, vol. 2, no. 3, pp. 564-571, 2011.

[7] G. Tsagarakis, R. C. Thomson, A. J. Collin and G. P. Harrison, "Assessment of the Cost and Environmental Impact of Residential Demand-Side Management," IEEE Transactions on Industry Applications, vol. 52, no. 3, pp. 2486-2495, 2016.

[8] S. Riaz, H. Marzooghi, G. Verbič, A. C. Chapman and D. J. Hill, "Generic Demand Model Considering the Impact of Prosumers for Future Grid Scenario Analysis," IEEE Transactions on Smart Grid, vol. 10, no. 1, pp. 819-829, 2019.

[9] S. Bahrami, M. H. Amini, M. Shafie-khah and J. P. S. Catalão, "A Decentralized Electricity Market Scheme Enabling Demand Response Deployment," IEEE Transactions on Power Systems, vol. 33, no. 4, pp. 4218-4227, 2018

[10] S. Martín, J. Pérez-Ruiz and P. López-Pérez, "Model to evaluate the system-wide impact of residential and commercial photovoltaic and storage units intended for self-consumption," IET Renewable Power Generation, vol. 13, no. 12, pp. 2111-2122, 2019.

[11] A. T. Procopiou, K. Petrou, L. F. Ochoa, T. Langstaff and J. Theunissen, "Adaptive Decentralized Control of Residential Storage in PV-Rich MV-LV Networks," IEEE Transactions on Power Systems, vol. 34, no. 3, pp. 2378-2389, 2019. 
[12] M. Carrion and J. Arroyo, "A Computationally Efficient Mixed-Integer Linear Formulation for the Thermal Unit Commitment Problem," IEEE Transactions on Power Systems, vol. 21, no. 3, pp. 1371-1378, 2006.

[13] D. Jaglal, W. J. Nacmanson and L. F. Ochoa, "Quantification and Verification of Residential Battery Response for Frequency Regulation in PV-Rich Power Systems," in IEEE PowerTech, Milano, 2019.

[14] W. J. Nacmanson, D. Jaglal and L. F. Ochoa, "Holistic Time-Varying Small Signal Stability Assessment in PV-Rich Power Systems," in IEEE PowerTech, Milano, 2019.

[15] NREL, "Renewable Electricity Futures Study," 2012.

[16] K. Selvakumar, G. Santhoshkumar and C. Sakthivel, "Optimal generation scheduling of thermal units with considering startup and shutdown ramp limits," in 2014 IEEE International Conference on
Computational Intelligence and Computing Research, Coimbatore, 2014.

[17] K. Van den Bergh and E. Delarue, "Cycling of conventional power plants: technical limits and actual costs," 2015.

[18] Agora Energiewende, "Flexibility in thermal power plants - With a focus on existing coal-fired power plants," 2017.

[19] Australian Government, Department of Industry and Science, "Australian Energy Statistics," 22 September 2015. [Online]. Available: https://data.gov.au/dataset/australian-energy-statistics. [Accessed 20 April 2018].

[20] "Australian PV market since April 2001," 2019. [Online]. Available: http://pv-map.apvi.org.au/analyses\#top. [Accessed 5 December 2018].

[21] Ausgrid, "Household Solar Power and Battery Survey," 2018. 